CORRIGENDUM

\section{Inhibitory control predicts language switching performance in trilingual speech production - CORRIGENDUM}

doi: 10.1017/S136672891100054X, Published by Cambridge University Press, 29 November 2011

Typographical errors occurred when preparing Table 2 and Table 5 of the paper by Linck et al. published online November 29, 2011. The raw reaction times reported in these tables were copied incorrectly and we now provide the corrected tables below. The text in the results section under the heading "Prior language effects", paragraph 2, should now read:

"Mean RTs for the nine conditions suggest that the prior language indeed affected the magnitude of switch costs (see Table 5). Specifically, L1 switch costs appear to be larger when switching from the less dominant L3. Switch costs in the L2 were larger when switching from the dominant L1 (263 $\mathrm{ms}$, on average) than from the less dominant L3 (163 ms, on average); similarly, L3 switch costs were numerically larger when switching from the dominant L1 than the L2."

The authors apologize for these errors.

Table 2. Correct reaction times (in $\mathrm{ms}$ ) and accuracy (in percent) for the picture-naming task, by Language and Condition.

\begin{tabular}{|c|c|c|c|}
\hline \multirow[b]{2}{*}{ Condition } & \multicolumn{3}{|c|}{ Language } \\
\hline & L1 & L2 & L3 \\
\hline & \multicolumn{3}{|c|}{ Reaction times } \\
\hline Switch & 1033 & 1187 & 1093 \\
\hline Non-switch & 826 & 974 & 986 \\
\hline \multirow[t]{2}{*}{ Switch Cost } & 206 & 212 & 107 \\
\hline & \multicolumn{3}{|c|}{ Accuracy } \\
\hline Switch & $(3.43)$ & $94.00 \quad(4.57)$ & $94.97 \quad(4.01)$ \\
\hline Non-switch & $(2.16)$ & $95.29 \quad(4.16)$ & $95.92 \quad(3.07)$ \\
\hline Switch Cost & 1.45 & 1.29 & 0.95 \\
\hline
\end{tabular}

L1 = native language (English); L2 = second language (French); L3 = third language (Spanish)

Notes: Reaction times reported as geometric means to ease interpretation, but analyses were conducted on log-transformed RTs. Accuracy standard deviations reported in parentheses. Bold indicates magnitude of switch costs to naming latencies or accuracy.
JARED A. LINCK

JOHN W. SCHWIETER

GRETCHEN SUNDERMAN

Table 5. Correct reaction times (in ms) and accuracy (in percent) for the picture-naming task, by preceding trial and current trial language.

\begin{tabular}{|c|c|c|c|c|c|c|}
\hline \multirow{2}{*}{$\begin{array}{l}\text { Preceding } \\
\text { trial }\end{array}$} & \multicolumn{6}{|c|}{ Current trial } \\
\hline & \multicolumn{2}{|l|}{$\mathrm{L} 1$} & \multicolumn{2}{|l|}{$\mathrm{L} 2$} & \multicolumn{2}{|l|}{ L3 } \\
\hline & \multicolumn{6}{|c|}{ Reaction times } \\
\hline L1 & \multicolumn{2}{|c|}{826} & \multicolumn{2}{|c|}{1238} & \multicolumn{2}{|c|}{1105} \\
\hline L2 & \multicolumn{2}{|c|}{1006} & \multicolumn{2}{|c|}{974} & \multicolumn{2}{|c|}{1081} \\
\hline \multirow[t]{2}{*}{ L3 } & \multicolumn{2}{|c|}{1060} & \multicolumn{2}{|c|}{1137} & \multicolumn{2}{|c|}{986} \\
\hline & & & Acc & Iracy & & \\
\hline L1 & 97.69 & $(2.16)$ & 93.39 & $(5.72)$ & 94.67 & (4.78) \\
\hline L2 & 95.76 & $(4.73)$ & 95.29 & (4.16) & 95.27 & (4.87) \\
\hline L3 & 96.73 & $(4.03)$ & 94.61 & (5.19) & 95.92 & (3.07) \\
\hline
\end{tabular}

L1 = native language (English); L2 = second language $($ French); L3 = third language (Spanish)

Notes: Reaction times reported as geometric means to ease interpretation, but analyses were conducted on log-transformed RTs. Accuracy standard deviations reported in parentheses.

\section{Reference}

Linck, J. A., Schwieter, J. W., \& Sunderman, G. Inhibitory control predicts language switching performance in trilingual speech production. Bilingualism: Language and Cognition. Published by Cambridge University Press, November 29, 2011. doi: 10.1017/S136672891100054X. 